ISSN 0103-5150

Fisioter. Mov., Curitiba, v. 24, n. 1, p. 147-154, jan./mar. 2011 Licenciado sob uma Licença Creative Commons

\title{
Existe associação entre doenças venosas e nível de atividade física em jovens?
}

\author{
Is there association between venous diseases and physical activity \\ level in young?
}

\section{Júlio César Oliveira Pena ${ }^{[a]}$, Luciana Bilitário Macedo ${ }^{[\mathrm{b}]}$}

[a] Fisioterapeuta pela União Metropolitana de Educação e Cultura (UNIME), Lauro de Freitas, Bahia - Brasil.

[b] Fisioterapeuta, Mestre em Medicina e Saúde Humana pela Escola Bahiana de Medicina e Saúde Pública, professora assistente na Escola Bahiana de Medicina e Saúde Pública, docente no curso de Fisioterapia da União Metropolitana de Educação e Cultura (UNIME), Lauro de Freitas, Bahia - Brasil, e-mail: lubilitario@ig.com.br

\section{Resumo}

Introdução: A associação dos benefícios da prática regular da atividade física a indicadores de saúde encontra-se amplamente discutida na literatura evidenciando a relação de um estilo de vida sedentário com as doenças crônicas degenerativas. Objetivo: Descrever a prevalência de doenças venosas entre jovens e sua associação com a atividade física. Materiais e métodos: Corte transversal. Amostra: jovens voluntários. Os indivíduos responderam a uma ficha clínica e foram examinados por meio do sistema de classificação clínica, etiológica, anatômica e patofisiológica (CEAP), que classifica a gravidade e a incapacidade para o trabalho de pessoas com doenças venosas; eles responderam também ao questionário internacional de atividade física (IPAQ). Resultados: Participaram 95 indivíduos, sendo 57,9\% (55) mulheres, tendo como média de idade 26,12 $\pm 4,5(18 \mapsto 35)$. De acordo com o IPAQ, os indivíduos foram classificados em categoria 1 (inativos): 41,1\%; categoria 2 (moderadamente ativos): 49,5\%; e categoria 3 (ativos): 9,5\%. Houve diferença entre os níveis de atividade física entre homens e mulheres ( $p=0,02)$. Na classificação da CEAP foram encontrados: classe $0=43,2 \%$; classe $1=45,3 \%$; classe $2=11,6 \%$, já as demais classes não apareceram na amostra. Mulheres apresentaram maior frequência de doenças vasculares que homens $(p<0,001)$. O maior nível de atividade física esteve associado com a menor prevalência de doenças venosas periféricas $(\mathrm{p}=0,02)$. Conclusões: Na amostra foi encontrada prevalência elevada de doenças venosas, maior entre mulheres. Foi elevado o número de indivíduos considerados sedentários pelo IPAQ, sendo os homens mais 
ativos quando comparados às mulheres. Os indivíduos com maior nível de atividade física apresentaram menor frequência de doenças venosas.

Palavras-chave: Insuficiência venosa crônica. Atividade física. Sedentarismo.

\section{Abstract}

Introduction: The association of the benefits of regular physical activity to health indicators is widely discussed in the literature demonstrating the relationship between a sedentary lifestyle and chronic diseases. Objective: To describe the prevalence of venous diseases among young person and their association with physical activity. Materials and methods: Cross-section. Sample: young volunteers. Individuals responded to a medical record and were examined through a system of clinical classification, etiologic, anatomic and pathophysiologic (CEAP) which classifies the severity and incapacity to work of people with venous disease, and responded to the international physical activity questionnaire (IPAQ ). Results: 95 subjects participated, 57.9\% (55) women. Mean age of $26.12 \pm 4.5$ (18-35), according to the IPAQ individuals were classified in category 1 (inactive): 41.1\%; category 2 (moderately active): $49.5 \%$; and category 3 (active): 9.5\%. Difference between physical activity levels between men and women $(p=0.02)$. In the CEAP classification were found: class $0=43.2 \%$; class $1=$ $45.3 \%$; class $2=11.6 \%$; and the other classes did not appear in the sample. Women had a higher frequency of vascular disease than men ( $p<0.001)$. Higher level of physical activity was associated with lower prevalence of peripheral venous disease ( $p=0.02)$. Conclusions: The sample was found high prevalence of venous disease was higher among women. It was the high number of individuals considered sedentary by IPAQ, men being more active when compared to women. Individuals with higher levels of physical activity had lower prevalence of venous diseases.

Keywords: Venous insufficiency. Physical activity. Sedentary lifestyle.

\section{Introdução}

A insuficiência venosa crônica (IVC) é definida como uma anormalidade do funcionamento do sistema venoso causada por uma incompetência valvular associada ou não à obstrução do fluxo venoso. Pode afetar o sistema venoso superficial, o sistema venoso profundo ou ambos. Além disso, a disfunção venosa pode ser resultado de uma desordem congênita ou adquirida (1).

Com o objetivo de uniformizar a literatura médica dos pacientes com IVC foi criado o sistema de classificação clínica, etiológica, anatômica e fisiopatológica (CEAP), que por meio de uma pontuação classifica a gravidade clínica e a incapacidade para o trabalho de pessoas com doenças vasculares $(2,3)$.

A IVC é uma doença comum na prática clínica e suas complicações, principalmente a úlcera de estase venosa, causam morbidade significativa. A ulceração afeta a produtividade no trabalho, gerando aposentadoria ou invalidez, além de restringir as atividades da vida diária e o lazer. Para muitos pacientes, as doenças venosas significam dor, perda da mobilidade funcional e piora na qualidade de vida (4).

No Brasil, a importância socioeconômica da IVC passou a ser considerada pelo governo somente nos últimos anos, o que tem levado ao aumento no interesse pelo conhecimento científico e clínico das questões relacionadas a essa doença (5).

0 sedentarismo é visto atualmente como problema mundial de saúde. Entre as razões que levam à inatividade, um dos possíveis fatores é o desconhecimento de como se exercitar, as finalidades de cada exercício, as limitações de alguns grupos populacionais e as percepções distorcidas em relação ao benefício do movimento $(6,7)$. 0 incremento da atividade física de uma população contribui decisivamente para a melhora da saúde pública, com forte impacto na redução dos custos com o tratamentos, inclusive hospitalares, de doenças relacionadas ao sedentarismo $(8,9)$.

0 exercício físico vem comprovando que é um grande aliado no tratamento das doenças vasculares periféricas (DVP). Os benefícios da atividade física na DVP estão principalmente relacionados à 
diminuição da claudicação intermitente e à melhora do metabolismo no músculo, favorecendo uma maior aderência das pessoas à prática supervisionada da atividade física (10). 0 exercício físico aumenta o tônus muscular dos membros inferiores e, consequentemente, pode melhorar sua ação no sistema venoso, com diminuição na pressão de deambulação e melhora do retorno venoso (11).

A meta deste trabalho foi estabelecer uma associação entre o nível de atividade física e o grau de insuficiência venosa periférica apresentado nessa população, bem como descrever a prevalência da DVP entre jovens.

\section{Materiais e métodos}

Estudo de corte transversal com uma amostra formada por 95 jovens estudantes da Faculdade de Ciências Agrárias da Saúde - União Metropolitana de Educação e Cultura (UNIME), em Lauro de Freitas, Bahia. Foram incluídos jovens de ambos os sexos, com idade maior que 18 anos. Foram excluídos indivíduos que apresentassem história clínica autorreferida de: doenças cardíacas; pneumopatias; hepatopatias; neoplasia; doenças musculoesqueléticas; e gestação.

\section{Aplicação do IPAQ e CEAP}

Todos os participantes fizeram o preenchimento de uma ficha clínica com dados sobre sexo, idade, uso de medicações, presença ou não de doenças crônicas, sintomatologia e fator hereditário para doenças venosas. A avaliação constou da aplicação da CEAP, sendo realizada por um único examinador, além da aplicação do IPAQ versão curta, que contém seis questões sobre atividades físicas de lazer, atividades domésticas, de transporte, de jardinagem e relacionadas ao trabalho. 0 questionário classifica os níveis de atividade física em categorias, como: um (atividade leve ou de caminhada); dois (atividade moderada); e três (muito ativos). Também foi calculado o escore contínuo por meio do gasto energético estimado pelos equivalentes metabólicos (METS) em METS/minuto/semana nas atividades de caminhada (categoria um), atividades moderadas (categoria dois) e atividades vigorosas (categoria três) (Anexo 1).
Análise estatística

As variáveis contínuas (idade e escore contínuo do IPAQ) foram expressas em média e desvio-padrão e, para comparação entre elas, foi utilizado o teste $t$ de Student. As variáveis categóricas foram descritas em frequência e a comparação entre as proporções foi feita pelo teste do Qui-quadrado.

\section{Aspectos éticos}

Este estudo obedeceu aos critérios da ética em pesquisa com seres humanos conforme a Resolução n. 196/96 do Conselho Nacional de Saúde, sendo aprovado pelo Comitê de Ética em Pesquisa da Faculdade de Tecnologia e Ciências sob o Parecer n. 01.156-2009.

\section{Resultados}

A amostra foi composta por 95 voluntários, tendo as mulheres correspondido a 57,9\% (55). A média de idade dos participantes foi de 26,12 anos $\pm 4,5$ $(18 \longmapsto 35)$. Dentre os participantes, 73,4\% referiram história familiar de varizes. Quando questionados sobre a sintomatologia apresentada em membros inferiores, 36,6\% referiram dores, 48,4\% relataram sensação de peso e cansaço em membros inferiores e 9,5\% descreveram "outras sensações". Em relação ao uso de meias elásticas, apenas 1,1\% dos participantes referiram fazer uso delas. A caracterização da amostra está demonstrada na Tabela 1.

Quando avaliada a frequência de doenças venosas entre os sexos, o número de mulheres com problemas vasculares na amostra foi de 48 e o dos homens foi 8 , com significância estatística $(p=0,02)$.

$\mathrm{Na}$ análise da prevalência de doenças vasculares, as mulheres foram mais acometidas do que os homens $(\mathrm{p}<0,01)$.

No que diz respeito à avaliação dos níveis de atividade física avaliados pelo IPAQ, 41,1\% eram sedentários; 49,5\%, moderadamente ativos; e 9,5\%, ativos. Homens foram considerados mais ativos do que as mulheres $(p=0,02)$. Já em relação à avaliação pela CEAP: $43,2 \%$ dos participantes pertenceram ao grupo de classificação clínica CEAP $=0$ (sem sintomas venosos); 45,3\% ao CEAP = 1 (com presença de telangiectasias) e 11,6\% ao CEAP $=2$ (com 
presença de veias varicosas). As demais classes não apareceram na amostra do estudo

A relação do nível de atividade física e do IVC demonstrou que o nível de indivíduos sedentários com IVC foi maior quando estes foram comparados aos indivíduos sedentários sem IVC ( $\mathrm{p}=0,02)$ (Figura 1).

Tabela 1 - Características clínicas, prevalência de IVC de acordo com a CEAP e nível de atividade física avaliado pelo IPAQ entre os jovens estudados*

\begin{tabular}{lc}
\hline $\mathbf{N}=\mathbf{9 5}$ & \\
\hline Idade & $26,12 \pm 4,5$ \\
Prevalência de IVC & $11,6 \%$ \\
História familiar de IVC & $73,4 \%$ \\
Meias elásticas & $1,1 \%$ \\
Ativos & $9,5 \%$ \\
Moderadamente ativos & $49,5 \%$ \\
Pouco ativos & $41,1 \%$ \\
\hline
\end{tabular}

Legenda: * = variáveis contínuas descritas em média e desviopadrão; variáveis categóricas mostradas em frequências; IVC = insuficiência venosa crônica.

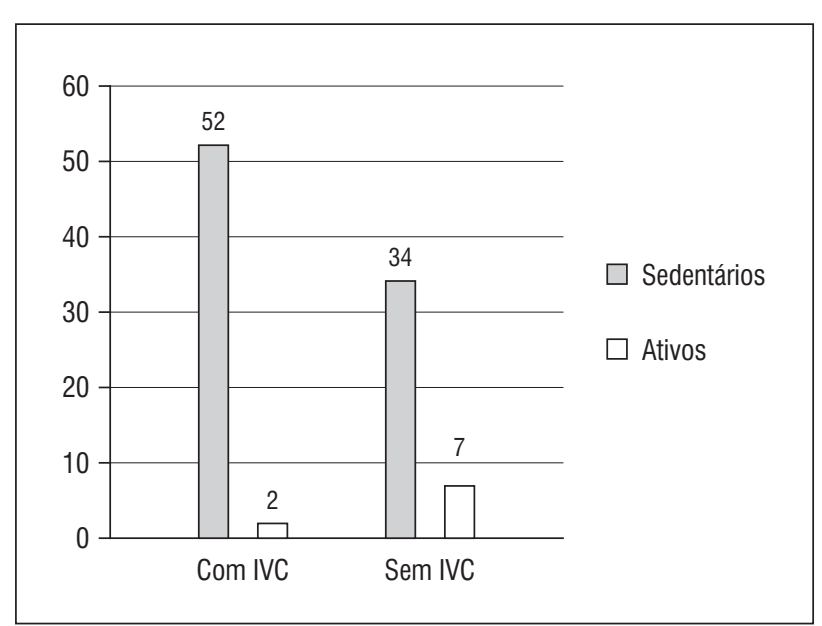

Figura 1 - Associação entre nível de atividade física e presença de doença venosa periférica avaliada pela CEAP entre jovens universitários com $p=0,02$

\section{Discussão}

De acordo com o presente estudo ficou evidenciado que as pessoas fisicamente mais ativas apresentavam menor frequência de doença venosa crônica, sendo esse um dado diferenciado na literatura, especialmente quando considerarmos a média de idade da população estudada.

Figueira Jr et al. (12) demonstraram que a prática de atividade física regular tem sido considerada como um dos fatores moduladores mais importantes na melhoria, na manutenção e na recuperação do nível de saúde populacional, na medida em que o sedentarismo tem forte relação com a prevalência de doenças crônico-degenerativas. Carvalho et al. (8) relataram, também, que o incremento da atividade física de uma população contribui decisivamente para a saúde pública, com impacto na redução dos custos com tratamentos, inclusive hospitalares, uma das razões de seus consideráveis benefícios sociais.

0 presente estudo verificou que os homens eram mais ativos fisicamente do que as mulheres. Costa et al. (13) analisaram 4.030 funcionários de uma universidade do Estado do Rio de Janeiro e obtiveram uma prevalência de sedentarismo de 47,8\% entre os homens e de $59,2 \%$ entre as mulheres. Isso também foi verificado no trabalho de Masson et al. (14), que, estudando o sedentarismo em mulheres adultas da cidade de São Leopoldo, Rio Grande do Sul, mostraram que 37\% eram sedentárias, 59,4\% insuficientemente ativas e somente $3,6 \%$ ativas.

França e Tavares (15) citam que a prevalência de IVC na população aumenta com a idade. Segundo Callam et al. (16), de $10 \%$ a $20 \%$ da população dos países ocidentais possui veias varicosas ou algum grau de IVC nos membros inferiores. Neste trabalho foi verificada uma prevalência de $11,6 \%$ de jovens com veias varicosas, similar aos demais citados.

A média de idade deste trabalho foi de 26,12 anos. Evans et al. (17) dizem que a partir da puberdade existe aumento progressivo da frequência de varizes, chegando a atingir mais de $70 \%$ das pessoas acima de 70 anos. Maffei (18) verificou que a incidência da IVC é mais alta a partir da terceira década de vida, atingindo o indivíduo em plena maturidade, quando sua capacidade de trabalho é maior.

De acordo com esta pesquisa, a população feminina possuiu maior frequência de IVC quando comparada ao sexo masculino. Alberti et al. (11), BeebeDimmer et al. (19) e Bergan et al. (20) mostraram maior prevalência de IVC na população feminina, justificando ser o sexo feminino um fator de risco para o desenvolvimento de IVC.

Nesta pesquisa, foi possível registrar a história familiar como um fator predisponente da presença 
de varizes. Bonamigo (21) relatou que a predisposição familiar é um dos fatores responsáveis pelas varizes primárias, estimando que aproximadamente $75 \%$ da varizes são determinadas geneticamente. Lee et al. (22) e Berard et al. (23) referem que o fator familiar é um dos principais componentes na etiologia das IVC, entretanto, genes específicos relacionados a ela não foram identificados.

De acordo com Bonamigo (21), indivíduos com doenças venosas crônicas podem queixar-se de sensação de peso ou cansaço, dores nas pernas, câimbras e edemas, já que esses sintomas são comuns entre os doentes com IVC. Neste trabalho esses sintomas também foram verificados. Alberti et al. (11) relatam que dores e sensação de desconforto nas pernas eram citadas com grande frequência pelos indivíduos com doenças venosas, entretanto, alguns doentes podem ser assintomáticos.

Os resultados deste trabalho demonstraram que uma minoria dos participantes fazia uso de meias de compressão elástica. Com relação ao uso dessas meias, França et al. (12) citam algumas indicações para seu uso, como: IVC moderada, avançada e pequenas veias varicosas. Figueiredo et al. (2) discutem que, apesar da terapia de compressão elástica necessitar de mais estudos para melhor descrever o seu mecanismo de ação, o seu uso na prática é grande. Contudo, essa terapia possui difícil avaliação quanto aos reais benefícios que pode proporcionar no tratamento da IVC.

As principais limitações desta pesquisa foram: o predomínio do gênero feminino na amostra; as limitações quanto à CEAP, que é a classificação mais utilizada para avaliar a IVC, sendo, no entanto, considerada complexa, de difícil abordagem e um examinador dependente. As limitações inerentes ao uso do IPAQ tais como o viés da memória ou lembrança e a variedade de procedimentos de escore, podem causar sobreposição de respostas de atividades de caminhada moderada e vigorosa.

\section{Conclusões}

$\mathrm{Na}$ amostra foi encontrada a prevalência de $11,6 \%$ de jovens com veias varicosas, sendo maior a frequência entre as mulheres. Foi elevado o número de indivíduos considerados sedentários pelo IPAQ sendo os homens mais ativos quando comparados às mulheres. Os indivíduos com maior nível de atividade física apresentaram menor frequência de doenças vasculares.

\section{Referências}

1. Porter JM, Moneta LG. Reporting standards in venous disease: an update. J Vasc Surg. 1995;21(4):635-45.

2. Figueiredo MAM, Filho AD, Cabral ALS. Avaliação do efeito da meia elástica na hemodinâmica venosa dos membros inferiores de pacientes com insuficiência venosa crônica. J Vasc Br. 2004;21(5):231-7.

3. Barros Jr NB. Insuficiência venosa crônica. J Vasc Br. 2003;21(5):1-7.

4. Oliveira RA, Barros Jr N, Miranda Jr F. A variabilidade hemodinâmica venosa detectada pelos parâmetros da pletismografia a ar nas classes clínicas da classificação. CEAP. J Vasc Br. 2007;6(4):359-65.

5. Maffei FHA, Lastória S,Yoshida WB. Doenças vasculares periféricas. 3a ed. Rio de Janeiro: Medsi; 2002. p. 1499-509.

6. Marani F, Oliveira AR, Omori MK. A atividade física em adolescentes de diferentes níveis sócio-econômicos. Rev Educ Fis UEM. 2005;16(1):67-71.

7. Domingues MR, Araújo CLP, Gigante DP. Conhecimento e percepção sobre exercício físico em uma população adulta urbana do sul do Brasil. Cad Saúde Pública. 2004;20(1):1-14.

8. Carvalho T, Nóbrega ACL, Lazzoli JK, Magni JRT, Rezende L, Drummond FA, et al. Posição oficial da Sociedade Brasileira de Medicina do Esporte: atividade física e saúde. Rev Bras Med Esporte. 1996;2(4):79-81.

9. Matsudo SMM, Matsudo VKR, Araújo TL, Andrade DR, Andrade EL, Oliveira LC, et al. Nível de atividade física da população do estado de São Paulo: análise de acordo com o gênero, idade, nível socioeconômico, distribuição geográfica e do conhecimento. Rev Bras Ciênc e Mov. 2002;10(4):41-50.

10. Silva DK, Nahas MV. Atividade física habitual e qualidade de vida relacionada à saúde em mulheres com doença vasculare periférica. Rev Bras Ciênc e Mov. 2004;12(40):63-8.

11. Alberti LR, Petroianu A, França DC, Silva TMF. Efeito da atividade física na insuficiência venosa crônica dos membros inferiores. Acta Med Port. 2008;21:215-20. 
12. Figueira Jr AJF. Potencial da mídia e tecnologias aplicadas no mecanismo de mudança de comportamento, através de programas de intervenção da atividade física. Rev Bras Ciênc e Mov. 2000;8(3):39-46.

13. Costa VB, Werneck GL, Lopes CS, Faerstein E. Associação entre fatores sócio-demográficos e prática de atividade física de lazer no estudo pró-saúde. Cad Saúde Pública. 2003;19(1):1095-105.

14. Masson CR, Dias-da-Costa JS, Olinto MTA, Meneghel S, da Costa CC, Bairros F, et al. Prevalência de sedentarismo nas mulheres adultas da cidade de São Leopoldo, Rio Grande do Sul, Brasil. Cad Saúde Pública. 2005;21(6):1685-94.

15. França LHG, Tavares V. Insuficiência venosa crônica. Uma atualização. J Vasc Br. 2003;2(4):318-28.

16. Callam MJ. Epidemiology of varicose veins. Br J Surg. 1994;81(2):1671-3.

17. Evans CJ, Fowkes FGR, Hajivassiliou CA, Harper DR, Ruckley CV. Epidemiology of varicose veins. Int Angiol. 1994;13(3):263-70.

18. Maffei FHA. Varizes dos membros inferiores: epidemiologia, etiolopatogenia e fisiopatologia. In: Maffei FHA, Lastória S, Yoshida WB, Editores. Doenças vasculares periféricas. 3a ed. Rio de Janeiro: Medsi; 2002. p. $1499-510$.
19. Beebe-Dimmer JL, Pfeifer JR, Engle JS, Schottenfeld D. The epidemiology of chronic venous insufficiency and varicose veins. Ann Epidemiol. 2005;15(3):175-84.

20. Bergan JJ, Schmid-Schönbein GW, Smith PD, Nicolaides AN, Boisseau MR, Eklof B. Chronic venous disease. N Engl J Med. 2006;355(5):488-98.

21. Bonamigo TP. Doenças vasculares: texto e atlas. Porto Alegre: Revinter; 2003. p. 264-271.

22. Lee AJ, Evans CJ, Allan PL, Ruckley CV, Fowkes FG. Lifestyle factors and the risk of varicose veins. J Clin Epidemiol. 2003;56(2):171-9.

23. Bérard A, Abenhaim L, Platt R, Kahn SR, Steinmetz O. Risk factor for the first-time development of venous ulcers of the lower limbs: the influence of heredity and physical activity. Angiology. 2002;53(6):647-57.

Recebido: 13/07/2010 Received: 07/13/2010

Aprovado: 05/12/2010 Approved: 12/05/2010 


\begin{tabular}{|c|c|c|}
\hline \multicolumn{3}{|c|}{ Anexos } \\
\hline \multicolumn{3}{|c|}{$\begin{array}{l}\text { Anexo I - Classificação clínica, etiológica, anatômica e fisiopatológica } \\
\text { (CEAP) para doenças venosas de membros inferiores (3) }\end{array}$} \\
\hline \multicolumn{3}{|c|}{ Classificação CEAP } \\
\hline Classe & Peso & Escore \\
\hline $0 / 1$ & Sintomas e/ou telangiectasias & 1 \\
\hline 2 & Veias varicosas & 5 \\
\hline 3 & Edema venoso & 10 \\
\hline 4 & Distúrbios tróficos & 20 \\
\hline 5 & Úlcera venosa cicatrizada & 50 \\
\hline 6 & Úlcera venosa ativa & 100 \\
\hline
\end{tabular}

Anexo II - Questionário internacional de atividade física versão curta (Ipaq)

Nós estamos interessados em saber que tipo de atividade física as pessoas fazem como parte do seu dia a dia. As perguntas estão relacionadas ao tempo que você gastou fazendo atividade física na última semana. Obrigado pela sua participação!

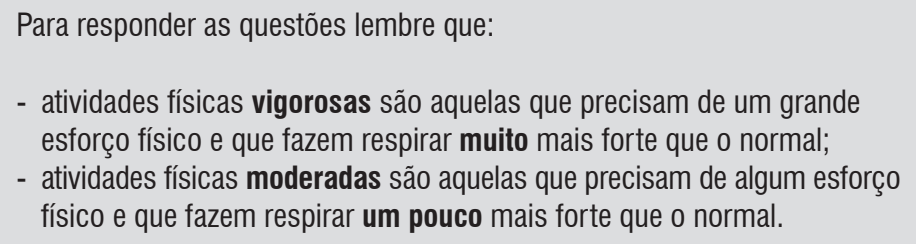

1a) Em quantos dias da última semana você caminhou por pelo menos 10 minutos contínuos, em casa ou no trabalho, como forma de transporte para ir de um lugar para outro, por lazer, por prazer ou como forma de exercício?
Dias por semana
( ) Nenhum

1b) Nos dias em que você caminhou por pelo menos 10 minutos contínuos, quanto tempo no total você gastou caminhando (por dia)?

Horas: ___ minutos:

2a) Em quantos dias da última semana você realizou atividades moderadas por pelo menos 10 minutos contínuos, como, por exemplo, pedalar leve na bicicleta; nadar; dançar; fazer ginástica aeróbica leve; jogar vôlei recreativo; carregar pesos leves; fazer serviços domésticos na casa, no quintal ou no jardim, como varrer, aspirar, cuidar do jardim; ou qualquer atividade que fez aumentar moderadamente sua respiração ou seus batimentos do coração (por favor não inclua caminhada).

Dias por semana ( ) Nenhum 
2b) Nos dias em que você fez essas atividades moderadas por pelo menos 10 minutos contínuos, quanto tempo no total você gastou fazendo essas atividades (por dia)?

Horas: , minutos:

3a) Em quantos dias da última semana você realizou atividades vigorosas por pelo menos 10 minutos contínuos, como, por exemplo, correr; fazer ginástica aeróbica; jogar futebol; pedalar rápido na bicicleta; jogar basquete; fazer serviços domésticos pesados em casa, no quintal ou cavoucar no jardim; carregar pesos elevados; ou qualquer atividade que fez aumentar muito sua respiração ou seus batimentos do coração.

Dias___ por semana ( ) Nenhum

3b) Nos dias em que você fez essas atividades vigorosas por pelo menos 10 minutos contínuos, quanto tempo no total você gastou fazendo essas atividades (por dia)?

Horas: , minutos:

Para preenchimento do pesquisador:

METS - min/semana:

Classificação: 